\title{
Analysis of optimum wire rope configuration for equal unidirectional torsional stiffness for flexible steering shaft
}

\author{
Najaf Hussain ${ }^{1}$, Masri Bin Baharom ${ }^{1, a}$ and Mui'nuddin Maharun ${ }^{1}$ \\ ${ }^{1}$ Department of Mechanical Engineering, Universiti Teknologi PETRONAS Malaysia
}

\begin{abstract}
The design and modeling of Low Stiffness Resilience Shaft (LSRS) for the Semi-Active Steering (SAS) system using wire ropes is discussed in this paper, along with the static structural torsion test simulation of the wire ropes in order to determine the best possible configuration which serves the purpose of an LSRS. The importance of this study arises due to the unidirectional torsional properties of a wire rope. For an effective operational LSRS, the wire ropes need to have similar angular deflection in both the clockwise and anti-clockwise direction. LSRS, an integral component of the SAS is a flexible shaft that can replace the conventional rigid shaft of the steering system and allows active control to be performed. 3D solid models of the simple strand and the 4 strand wire ropes used in finite element analysis were generated in CAD software SolidWorksTM. The single strand and the different configuration of wire ropes required to function the LSRS effectively were then analyzed using Finite element simulation in ANSYS $\mathrm{S}_{\mathrm{TM}}$. A single wire rope could not be used because its construction has inconsistency in the torsional stiffness in clockwise and anti-clockwise direction. The single-strand right-direction lay wire rope is found to have $16.05 \%$ angular deflection percentage difference in the clockwise and anticlockwise directions which indicates that using a single strand wire rope for the LSRS will cause the vehicle to have a variable response in the clockwise and anti clockwise direction upon turning the steering wheel. Due to this inconsistency, two variations namely Variation 1 and Variation 2 with arrangement of 4 strand wire rope were devised so that the angular deflection percentage difference would be negligible. Simulation results indicated that Variation 1 of the two variations with an angular deflection percentage difference of $0.34 \%$ in the clockwise and anti-clockwise direction respectively is best suited for the use in LSRS as it has almost negligible angular deflection percentage difference and will allow the vehicle to have similar steering response in the clockwise and anti-clockwise direction.
\end{abstract}

Keywords: Steering; Automotive; flexible shaft; wire rope; Finite Element simulation

\section{Introduction}

It has been more than 100 years since the introduction of the first automobile and the engineers have been able to make amazing technological advancements in the automotive field since then. For the need of drivers or passengers, engineers have been constantly improving the design of various systems of vehicles such as improved ride comfort, different suspension set ups and various tire profiles. With the increasing speeds of vehicles, safety became one of the important issues and therefore measures were taken to increase the safety of the drivers and passengers of moving vehicles, such as new and improved chassis designs. One of the examples is the case with the development of the steering system[1].

Drivers of vehicles were having difficulty to steer vehicles during parking at low speed maneuvers 50 years after the invention of automobile. With the increase in travelling speeds of automobiles, drivers were also looking for increased handling stability at high speeds. Furthermore, the need for higher power and speed led to the creation of heavier engines and chassis of the vehicles which meant that the rack loads increased and the drivers needed a lot of strength to turn the steering wheels [2]. In order to solve these problems, automotive engineers have come up with different types of steering systems such as Steer-by-Wire (SBW) steering system or the SAS system. SBW steering systems allow the amount of steering wheel operation to be transmitted in the form of electric signals to the vehicle wheels. These systems help improve control performance for vehicle safety while increasing vehicle design freedom [3]. One another design concept in pursuit of solving these problems is the Semi-Active-Steering (SAS) system technology [4] which takes care of all these problems, and provides advantages such as the Active control, ease of packaging and improved safety to the drivers.

The SAS system is like a SBW steering system but instead of a backup system there is a permanent connection between the steering wheel and the rack and pinion. The problem arises because current steering shafts cannot be used in this system as they are torsionally rigid

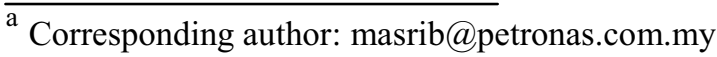


and will affect the SBW steering capabilities when the SBW system is active and will not allow active steering to be performed on the vehicle. Furthermore the conventional steering shaft is rigid along the axis, which may cause injury to the driver in case of a front end collision. Therefore in the SAS instead of a rigid shaft, there is a Low-stiffness-resilient-shaft (LSRS) which introduces active control to the vehicle [4]. The LSRS is the integral component of the SAS system and is always connected to the system unlike the (SBW) where the clutch mechanism is only engaged when the electric system fails. The properties of the LSRS should be as such that if the SBW system is still engaged, the LSRS doesn't affect the steering capability, but if the SBW system fails the LSRS should be torsionally rigid enough to provide the vehicle steering capabilities so that the vehicle can be maneuvered to safety. Furthermore, the LSRS should be flexible along its axis so that in case of an accident or a front end collision, the LSRS buckles and the driver isn't harmed. A suitable arrangement of wire ropes supported by bearings can give the properties needed to make a LSRS for an SAS system.

Wire rope is a versatile, flexible, high-strength member that is used in many mechanical systems to provide excellent tensile strength while it remains flexible. It is also used in power transmission application components which are physically separated or not colinear. Wire rope is typically classified by four parameters: (1) nominal diameter; (2) rope construction; (3) core type; and (4) breaking load. Variations in the make of the wire rope affect physical properties, such as flexibility, rupture strength, and service life [5].

The mechanical analysis of a wire rope structure is made complex by contact friction interactions taking place between elementary wires [6]. Similarly the analytical solutions for the torsional or tensile stresses of the wire rope normally neglect the frictional and contact effects. With the use of solid modeling and finite element analysis in a computer environment, it is possible to consider frictional, contact and other working conditions [7]. Finite element predictions of rope properties started in the early 1970s but it was restricted at the time. The developments in finite element software as well as in computer technology in respect to contact and model size in the recent years have made it much more feasible not only from a scientific but also from a practical point of view [8].

Because of the lay pattern of a single strand wire rope, it cannot be used for the construction of the LSRS as it will have varying angular deflection in the clockwise and anti-clockwise direction. The author models and performs simulation on the single strand wire rope to find out the angular deflection upon application of torque in both directions. Two different arrangements of wire ropes are then modeled so that the angular deflection in both directions is similar. FEA simulations are performed on the $3 \mathrm{D}$ solid models in $\mathrm{ANSYS}^{\mathrm{TM}}$ and the angular deflection is noted.

\section{System Concept Design}

The system setup of the SAS is shown in Figure 1. The LSRS, Power motor, Reaction motor, sensors and controllers are the parts and parcels of the system. The presence of the LSRS makes this system different from any other steering system in the automotive industry. The LSRS is basically used instead of a rigid steering shaft because firstly it offers a packaging advantage as it can allow the steering wheel to be placed on either side of the dashboard. Secondly, in case of a head on collision it will buckle. The LSRS can be made out of a cable which is flexible and resilient to the torque acting along its length. The main concern is the rigidity and stiffness of the cable so that when the main electric system fails, the driver should be able to steer the vehicle safely using just the LSRS.

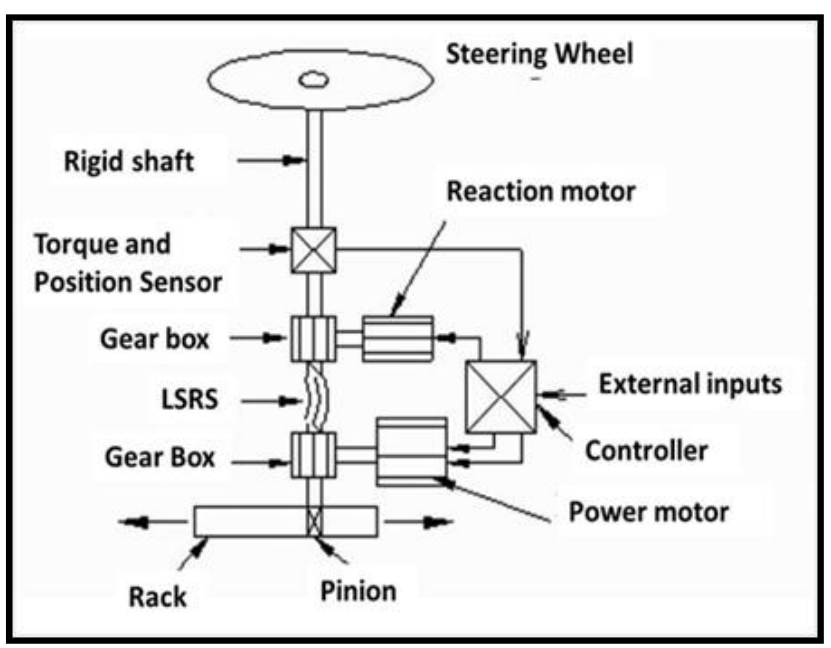

Figure 1. Schematic diagram of the SAS [4]

\subsection{Basic Configuration of the LSRS}

The proposed LSRS design mainly constitutes of two important components which are the bearings and wire ropes as shown in Figure 2(a).

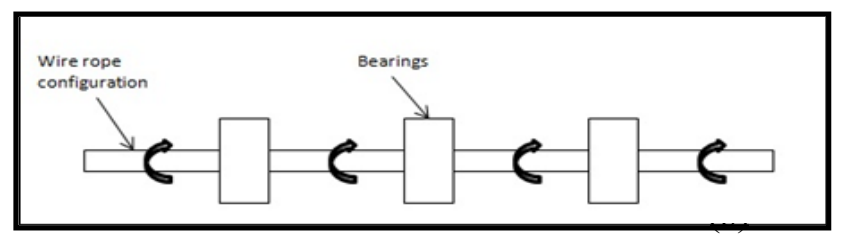

Figure 2(a). Basic Layout of the LSRS

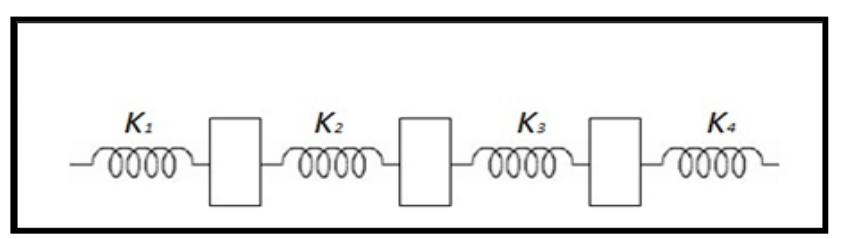

Figure 2(b). Wire rope sections denoted by their spring constants

Figure 2(a) shows the basic layout of the LSRS while Figure 2(b) denotes the wire rope sections as their spring constant. The main properties concerned with LSRS that affect the SAS are its torsional stiffness and axial flexibility which are determined by two factors namely: 
-The distance between the bearings.

- The configuration/arrangement of the wire rope.

The LSRS should be designed in such a way that a vehicle should be steered comfortably to safety in the event of a failure of the SBW system, meaning that the LSRS should be torsionally rigid enough to provide control to the car in the event of failure of the SBW but flexible so that active steering can be performed when the SBW system is engaged. The torsional stiffness is characterized by the spring constant or the torsion coefficient. The formula for the equivalent spring constant depends on the number of wire rope sections and can be derived from the Figure 2(b).

$$
\frac{1}{K_{e q}}=\frac{1}{K_{1}}+\frac{1}{K_{2}}+\frac{1}{K_{3}}+\ldots \ldots
$$

The unit for the Keq is $\mathrm{Nm} / \mathrm{rad}$. Hence the equivalent Torque on the LSRS can be calculated using the following equation:

$$
\tau=K_{e q} \theta
$$

Where $\tau$, is the torque exerted on the LSRS and $\theta$ is the angular deflection made by the LSRS in rad. The negative sign indicates that the direction of torque is opposite to the direction of twist.

\subsection{Configuration of the Wire Ropes in the LSRS}

The configuration of the wire rope is also of grave importance in designing the LSRS. The individual wires in a wire rope are organized in a circular pattern but are wound in a certain direction, either clockwise or anti clockwise. The wire ropes used to transmit torque are categorized under uni-directional or bi-directional. The uni-directional wire ropes are wound for one direction only and they can transmit torque in just one direction efficiently. Although bi-directional ropes can transmit torque in both directions, they have varying torsional stiffness because of the variable laying patterns of the wire ropes. The varying torsional stiffness values of the wire ropes is the reason why the configuration of the wire ropes is very important for the LSRS.

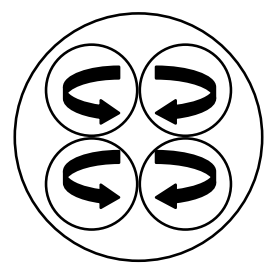

(a)

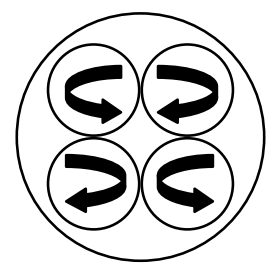

(b)
Figure 3. Arrangement of wire ropes for (a) Variation 1 (b) Variation 2

Two different configurations of the wire ropes were modeled and analyzed using finite element analyses (FEA) in ANSYS Workbench 15.0 in order to determine the best possible configuration which has similar torsional stiffness in clockwise and anti-clockwise directions. Figure 3(a) shows Variation 1, in which a simple arrangement of 4 strand wire ropes has been chosen. In this variation, two of the adjacent wire ropes are right-hand lay and the other two are left-hand lay so that the overall arrangement has similar stiffness, Keq in both directions. Variation 2 on the other hand, as shown in Figure 3(b), has two alternate wire ropes of right-hand lay and the other two alternate wire ropes of left-hand lay for similar purposes.

\section{MODELING OF THE WIRE ROPE}

This section discusses the modeling of the $1 \times 7$ wire rope strand and the complete wire rope model. The $1 \times 7$ is the basic strand construction which contains 7 individual wires wrapped in a right or left lay direction. Since there are many parameters which can be varied in the construction of a rope strand, it is important to have a general and accurate model which can predict the effects of possible variations of these parameters on the performance of the strand [9]. The solid models of the right and left lay wire ropes were developed and used to perform the simulations in ANSYSTM, so that the behavior of the wire rope at the application of torque could be studied.

The selected wire rope was a $1 \times 7$ strand made out of Galvanized steel in a nominal diametric size of 3.56 $\mathrm{mm}$. Table 1 shows the geometrical parameters of the simple strand.

Table 1. Geometric parameters of the $1 \times 7$ strand wire rope

\begin{tabular}{|c|c|}
\hline $\begin{array}{c}\text { Geometrical } \\
\text { parameters }\end{array}$ & Value \\
\hline Strand Diameter $(d)$ & $3.56 \mathrm{~mm}$ \\
\hline $\begin{array}{c}\text { Center wire diameter } \\
\left(2 R_{l}\right)\end{array}$ & $1.20 \mathrm{~mm}$ \\
\hline $\begin{array}{c}\text { Outer wire diameter } \\
\left(2 R_{2}\right)\end{array}$ & $1.18 \mathrm{~mm}$ \\
\hline $\begin{array}{c}\text { Pitch length or Lay } \\
\text { length }(p)\end{array}$ & $54.3 \mathrm{~mm}$ \\
\hline
\end{tabular}

For the current $1 \times 7$ strand, the outer wire helix radius and angle from Equation (3) and Equation (4) can be found out to be,

$$
\begin{gathered}
R=R_{1}+R_{2}=1.19 \mathrm{~mm} \\
\tan \alpha=\frac{p}{2 \pi R}=\frac{54.3}{2 \pi 1.19}=7.26 \\
\alpha=\mathbf{8 2 . 1 5 ^ { \circ }}
\end{gathered}
$$

The simple strand model of the above mentioned geometrical parameters is shown in Figure 4(a). Meanwhile Figure 4(b) shows the solid model with torque handles in order to make it easier to apply boundary conditions to the wire rope model. The two different types of variations were also modeled in order to analyze which variation is better in terms of equality of torsional stiffness in both directions, which can be seen in Figure 4(c) and Figure 4(d). 


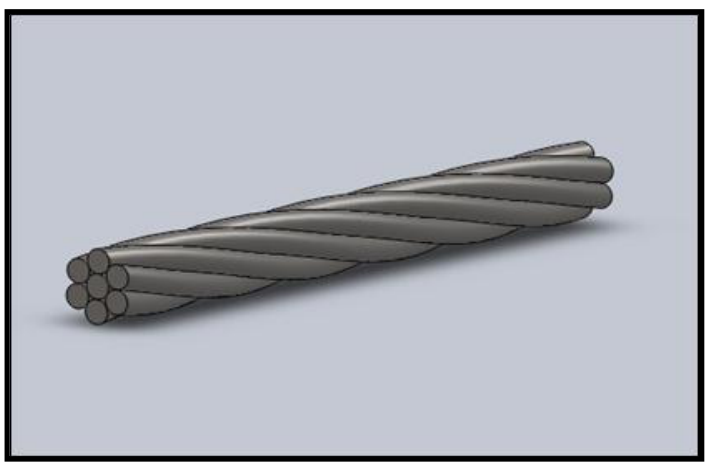

Figure 4(a). Solid model of the 1-strand wire rope

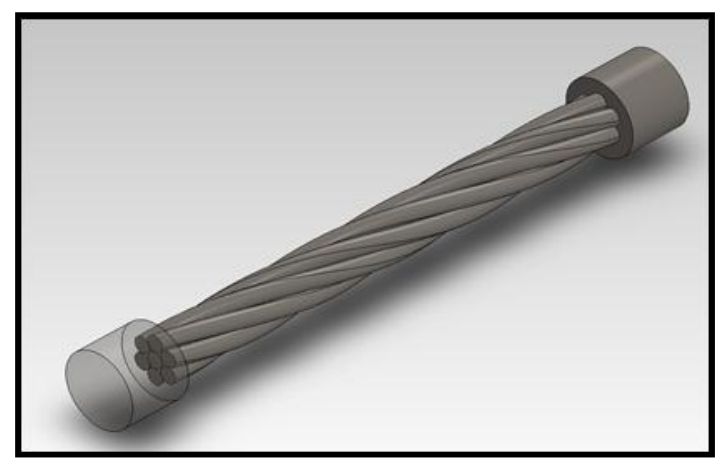

Figure 4(b). Solid model of the 1-strand wire rope with torque handles

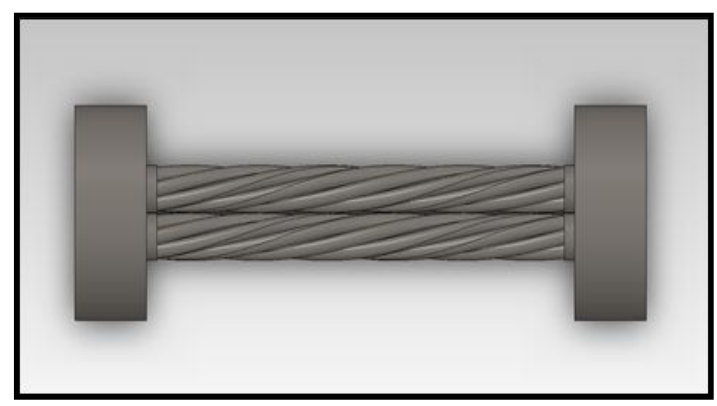

Figure 4(c). Top view of Variation 1 - adjacent wire ropes in same lay direction

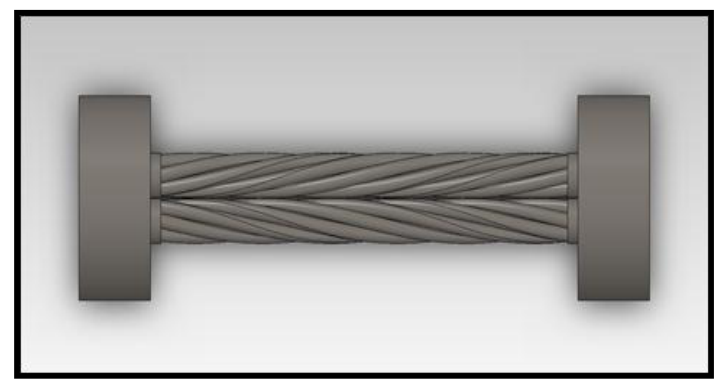

Figure 4(d). Top view of Variation 2 - alternate wire ropes in the same lay direction

\section{SIMULATION AND RESULTS}

\subsection{Simulation of the 1-strand wire rope}

In order to find the angle of deformation made by the wire rope at the application of a certain torque in both directions, static structural torsion test was performed on the 1-strand wire rope. The only available wire rope for this experiment is the right-lay direction. For the analysis, the torque was applied both in clockwise and anticlockwise directions in order to determine the difference in behavior of the wire rope in both directions. The frictional contact coefficient between individual wires was 0.115 [10]. Figure 5(a) shows the frictional contact between two individual wires of the wire rope.

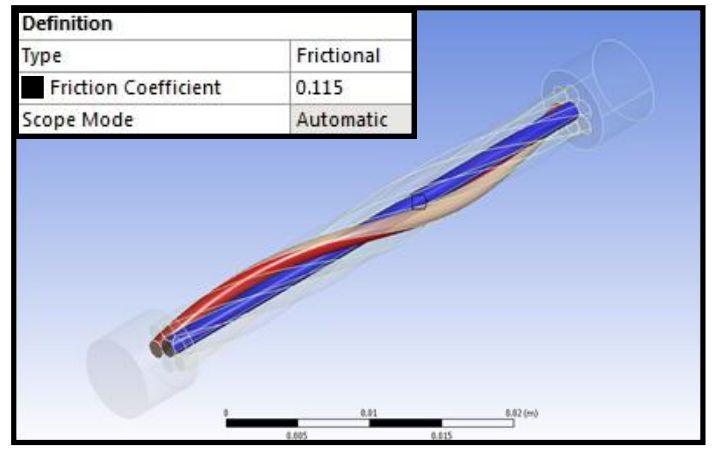

Figure 5(a). Frictional contact between the core and outer wire

Face Sizing was applied to every individual wire, in order to obtain a uniform mesh. The material assigned to the model was Galvanized steel as the purchased wire rope for the experiment was made of the same material. For the boundary conditions, one end of the wire rope was fixed and not allowed to displace in the $\mathrm{x}, \mathrm{y}$ or $\mathrm{z}$ directions and on the other end a torque of $0.05 \mathrm{~N} . \mathrm{m}$ magnitudes was applied. The results were then analyzed and compared. The total deformation for the anticlockwise and clockwise directions as a result of the applied torque can be seen in Figure 5(b) and 5(c) respectively.

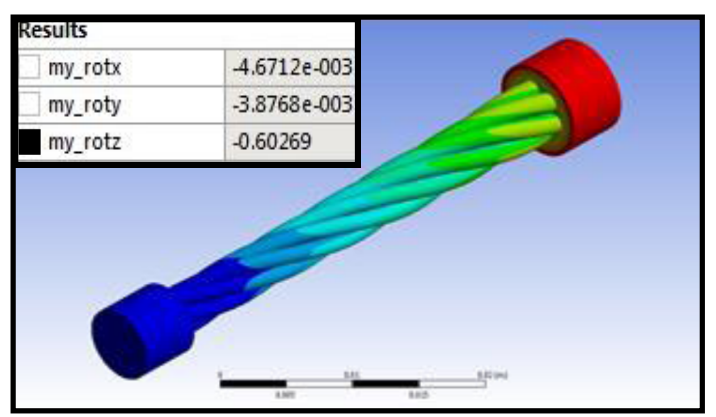

Figure 5(b). Angular deformation in degrees by application of Anti-clockwise torque

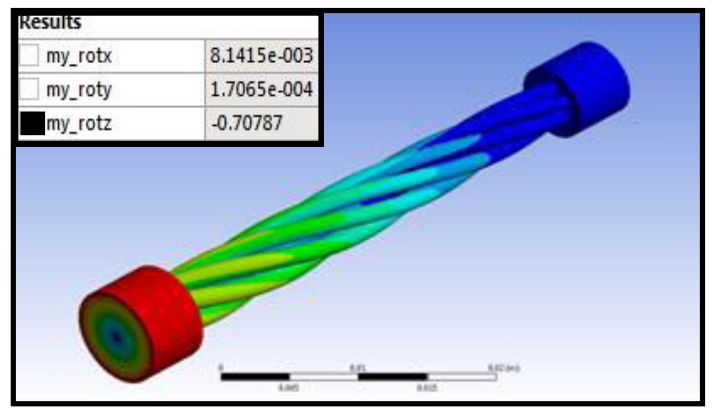

Figure 5(c). Angular deformation in degrees by application of Clockwise torque

A vast difference in the angular deflection is observed at the application of torque in the clockwise and anti-clockwise direction. Table 2 elucidates the angular 
deflections in degrees which are observed through the simulation results.

Table 2. Angular deflection and percentage difference of 1 strand

\begin{tabular}{|c|c|}
\hline \multicolumn{2}{|c|}{ Angular Deflection } \\
\hline \multirow{2}{*}{$\begin{array}{c}\text { Clockwise } \\
\text { deflection }\end{array}$} & $0.708^{\circ}$ \\
\cline { 2 - 2 } & $0.012 \mathrm{rads}$ \\
\hline $\begin{array}{c}\text { Anticlockwise } \\
\text { deflection }\end{array}$ & $0.603^{\circ}$ \\
\cline { 2 - 2 } $\begin{array}{c}\text { Percentage } \\
\text { difference }\end{array}$ & $0.010 \mathrm{rads}$ \\
\hline
\end{tabular}

An angular deflection of 0.708 degrees or $0.012 \mathrm{rad}$ is found in the clockwise direction and a deflection of 0.603 degrees or $0.010 \mathrm{rad}$ is noted in the anti- clockwise direction. The observed percentage difference is $16.05 \%$ which proves that the torsional stiffness in both directions varies hugely. The torsional stiffness can be calculated using Equation (5):

$$
K=\tau / \theta
$$

For clockwise direction:

$$
K=4.04 \mathrm{~N} . \mathrm{m} / \mathrm{rad}
$$

For anti-clockwise direction:

$$
K=4.75 \mathrm{~N} . \mathrm{m} / \mathrm{rad}
$$

The reason for the huge difference in the torsional stiffness as discussed earlier was due to the lay direction of the wire rope. The wire rope under consideration is right-hand lay and the application of the torque in the anti-clockwise direction will make it tighter and hence it will have higher stiffness in that direction. If the torque is applied in the clockwise direction, the torsional stiffness will be lower as the application of torque in that direction will cause the wire rope to un-wind. Because of these reasons, Variation 1 and Variation 2 were subjected to static structural torsion simulation.

\subsection{Simulation of the 4-strand wire rope arrangement}

To counter the torsional behavioral inconsistency of a single wire rope, two different variations of different arrangement of wire rope were simulated in ANSYSTM. The simulation procedure for the different variations was similar to the ones conducted on the 1-strand wire rope i.e. one end was fixed and the other end was allowed to rotate at a fixed torque. The torque applied on one end of both variations was 0.1 N.m.

The simulation results indicate a vast improvement in the angular deflection for both Variation 1 and 2 (see Table 3). The observed angular deflection in clockwise and anticlockwise direction is much more consistent and similar in values. Table 3 shows the Angular deflection in degrees and the percentage differences for both Variation 1 and Variation 2.
Table 3. Angular deflection and percentage difference of Variation 1 and Variation 2

\begin{tabular}{|c|c|c|}
\hline \multicolumn{3}{|c|}{ Angular Deflection } \\
\hline $\begin{array}{c}\text { Variation 1 Clockwise } \\
\text { deflection }\end{array}$ & 0.3580 & $\begin{array}{c}0.00625 \\
\mathrm{rad}\end{array}$ \\
\hline $\begin{array}{c}\text { Variation 1 } \\
\text { deflection }\end{array}$ & $0.357 \mathrm{o}$ & $\begin{array}{c}0.00623 \\
\mathrm{rad}\end{array}$ \\
\hline Percentage difference & 0.3460 & $\begin{array}{c}0.00603 \\
\mathrm{rad}\end{array}$ \\
\hline $\begin{array}{c}\text { Variation 2 Clockwise } \\
\text { deflection }\end{array}$ & $0.359 \mathrm{o}$ & $\begin{array}{c}0.00627 \\
\mathrm{rad}\end{array}$ \\
\hline $\begin{array}{c}\text { Variation 2 } \\
\text { Anticlockwise } \\
\text { deflection }\end{array}$ & $0.90 \%$ \\
\hline Percentage difference & \multicolumn{2}{|c|}{$3.90 \%$} \\
\hline
\end{tabular}

For Variation 1, the clockwise angular deflection is found to be 0.358 degrees and the anticlockwise deflection is 0.357 degrees. The percentage difference between the two was calculated to be $0.34 \%$. Similarly, for the Variation 2, the Clockwise deflection is 0.346 degrees and the Anti-clockwise deflection is 0.359 degrees with a percentage difference of $3.90 \%$. The torsional stiffness for the different Variations is calculated below using Equation (5).

-Variation 1

For clockwise direction: $K=15.99 \mathrm{~N} . \mathrm{m} / \mathrm{rad}$

For anti-clockwise direction: $K=16.04 \mathrm{~N} . \mathrm{m} / \mathrm{rad}$

-Variation 2

For clockwise direction: $K=16.58 \mathrm{~N} . \mathrm{m} / \mathrm{rad}$

For anti-clockwise direction: $K=15.94 \mathrm{~N} . \mathrm{m} / \mathrm{rad}$

The values of the coefficient of torsional stiffness indicate that these two variations have a higher value and indeed much stiffer than the 1-strand wire rope due to the introduction of 3 more wire ropes. The lower values of percentage difference indicate that the torsional inconsistency problem for the 1-strand can be overcome using the two suggested variations, especially Variation 1 with such a low percentage difference of $0.34 \%$ compared to the percentage difference of 1-strand wire rope which was found out to be $16.05 \%$.

\section{CONCLUSION}

The construction of the LSRS and the best configuration of the wire ropes required for its construction have been discussed in this paper. The use of wire ropes for the LSRS of the SAS system serves the purpose of allowing Active steering to be performed on the system because of its torsional flexibility, as well as making it safe in terms of a head on collision or beneficial in terms of packaging due to the property of it being axially flexible.

The simulations performed on the single strand wire rope indicated that there is a vast torsional inconsistency 
due to its construction as it is laid in the right lay direction. An angular deflection percentage difference of $16.05 \%$ was calculated in the clockwise and anticlockwise direction which indicates that a single wire rope cannot be used for the construction of the LSRS as it will cause the vehicle to have a variable response in the clockwise and anti-clockwise direction when the steering wheel is turned. Using this as the background, two different configurations of wire rope arrangements namely Variation 1 and Variation 2 were also simulated using similar boundary conditions. Variation 1, with an angular deflection percentage difference of $0.34 \%$ compared to the $3.90 \%$ of Variation 2 is considered suitable to be used for the construction of the LSRS.

\section{REFERENCES}

[1] Zhai P. Bilateral control of steer-by-wire vehicles. School of Electrical, Computer and Telecommunications Engineering, University of Wollongong. 2013.

[2] Holt DJ. Electric steering. a revolution in steering technology. Society of Automotive Engineers. 2002.

[3] Mogi K, Sugai T, Sakurai R, Suzuki N. Development of a new steer-by-wire system. NTN Technical Review No.79. 2011.

[4] Baharom MB, Hussain K, Day AJ. Design concepts and analysis of a semi- active-steering system for a passenger car. Proc. IMechE. 2009;223:283-292.

[5] Reid JD, Lechtenberg KA, Stolle CS. Development of advanced finite element material models for cable barrier wire rope. University of Nebraska, Lincoln. 2010.

[6] Otano N, Durville D, Usabiaga H. Finite element simulation of the mechanical behaviour of wire ropes, comparison with analytical models and experimental tests. 5th European Conference on Computational Mechanics (ECCM V). Barcelona, Spain. 2014.

[7] Gerdemeli I, Kurt S, Anıl AS. Fatigue life analysis of wire rope strands with finite element method. Key Engineering Materials. 2014;572: 513-516.

[8] Bechtold M. Modeling of steel ropes. 2009 SIMULIA Customer Conference. 2009.

[9] Jiang WG, Yao MS, Walton JM. A concise finite element model for simple straight wire rope strand. International Journal of Mechanical Sciences 41. 1999; 41:143-161.

[10] Jiang WG, Henshall JL. The analysis of termination effects in wire strand using finite element method. Journal of Strain Analysis. 1999;34:31-38.

\section{Notations}

$K \quad$ Spring constant/ Torsion constant (Nm/rad)

Keq Equivalent torsion constant of the LSRS (Nm/rad)

$\tau \quad$ Torque exerted by the LSRS (Nm)

$\theta$ Angle of twist from the equilibrium position (Rad)

$d \quad$ Strand diameter ( $\mathrm{mm})$

$R \quad$ Radius of the helix on the outer wire $(\mathrm{mm})$

$R_{l} \quad$ Radius of the center wire $(\mathrm{mm})$

$R_{2} \quad$ Radius of the outer wire $(\mathrm{mm})$
Pitch or Lay length of the wire rope (mm)

$\alpha \quad$ Helix angle of the outer wire (degrees) 